\title{
Noise perturbations in the Brownian motion and quantum dynamics
}

\author{
Piotr Garbaczewski \\ Institute of Physics, Pedagogical University, \\ pl. Słowiański 6, PL-65 069 Zielona Góra, Poland* \\ June 16, 2017
}

\begin{abstract}
The third Newton law for mean velocity fields is utilised to generate anomalous (enhanced) or non-dispersive diffusion-type processes which, in particular, can be interpreted as a probabilistic counterpart of the Schrödinger picture quantum dynamics.
\end{abstract}

If we consider a fluid in thermal equilibrium as the noise carrier, a kinetic theory viewpoint amounts to visualizing the constituent molecules that collide not only with each other but also with the tagged (colloidal) particle, so enforcing and maintaining its observed erratic motion. The Smoluchowski approximation takes us away from those kinetic theory intuitions by projecting the phase-space theory of random motions into its configuration space

*Email: pgar@omega.im.wsp.zgora.pl 
image which is a spatial Markovian diffusion process, whose formal infinitesimal encoding reads:

$$
d \vec{X}(t)=\frac{\vec{F}}{m \beta} d t+\sqrt{2 D} d \vec{W}(t)
$$

In the above $m$ stands for the mass of a diffusing particle, $\beta$ is a friction parameter, D is a diffusion constant and $\vec{W}(t)$ is a normalised Wiener process. The Smoluchowski forward drift can be traced back to a presumed selective action of the external force $\vec{F}=-\vec{\nabla} V$ on the Brownian particle that has a negligible effect on the thermal bath but in view of frictional resistance imparts to a particle the mean velocity $\vec{F} / m \beta$ on the $\beta^{-1}$ time scale, [1] . The noise carrier (fluid in the present considerations) statistically remains in the state of rest, with no intrinsic mean flows.

An implicit phase-space scenario of the Brownian motion refers to minute acceleration/deceleration events which modify (infinitely gently, [1], at a generic rate of $10^{21}$ times per second) velocities of realistic particles. Clearly, the microscopic energy-momentum conservation laws need to be respected in each separate collision event. In contrast to derivations based on the Boltzmann equation, this feature is completely alien to the Brownian motion theory. That energy-momentum deficit is one of "forgotten" or tacitly "overlooked" problems in the standard theory of the Brownian motion, see e.g. [2, 3].

An issue of origins of general isothermal flows in the noisy environment is far from being settled, [3]. Namely, the standard theory does not account (or refers to phenomena for which that is not necessary) for generic perturbations of the random medium as a reaction to the enforced (exclusively due to the impacts coming from the medium) particle motion. An ability of the medium to perform work i. e. to give a kinetic energy to Brownian particles (strictly speaking, in the local mean, hence on the ensemble average) appears to be a universal feature of the thermal bath even in the absence of any external force. 
Then, there arise problems with the thermal equilibrium, [3]. The tagged particle always propagates "at the expense" of the bath, which nonetheless remains "close" to its thermal equilibrium. In view of the involved local cooling and heating phanomena, which are necessary to keep in conformity with the second law of thermodynamics, the bath should develop local flows and thus in turn actively react back to what is being happenning to the particle in the course of its random propagation. Such environmental reaction, while interpreted as a feedback effect, surely can be neglected in an individual particle propagation sample. However, an obvious Brownian particle energymomentum "deficit" on the ensemble average (kinetic energy and momentum is carried locally by diffusion currents), indicates that the medium reaction may give observable contributions on the ensemble average, as a statistically accumulated feature. For a statistical ensemble of weakly-out-of-equilibrium systems, an effective isothermal scenario can be used again, but then mean flows in the noise carrier are "born" as a consequence of the averaging, [3].

It is well known that a spatial diffusion (Smoluchowski) approximation of the phase-space process, allows to reduce the number of independent local conservation laws (cf. [5]-8]) to two only. Therefore the Fokker-Planck equation can always be supplemented by another (independent) partial differential equation to form a closed system.

If we assign a probability density $\rho_{0}(\vec{x})$ with which the initial data $\vec{x}_{0}=$ $\vec{X}(0)$ for Eq. (1) are distributed, then the emergent Fick law would reveal a statistical tendency of particles to flow away from higher probability residence areas. This feature is encoded in the corresponding Fokker-Planck equation:

$$
\partial_{t} \rho=-\vec{\nabla} \cdot(\vec{v} \rho)=-\vec{\nabla} \cdot\left[\left(\frac{\vec{F}}{m \beta}-D \frac{\vec{\nabla} \rho}{\rho}\right) \rho\right]
$$

where a diffusion current velocity is $\vec{v}(\vec{x}, t)=\vec{b}(\vec{x}, t)-D \frac{\vec{\nabla} \rho(\vec{x}, t)}{\rho(\vec{x}, t)}$ while the forward drift reads $\vec{b}(\vec{x}, t)=\frac{\vec{F}}{m \beta}$. Clearly, the local diffusion current (a local flow that might be experimentally observed for a cloud of suspended particles 
in a liquid) $\vec{j}=\vec{v} \rho$ gives rise to a non-negligible matter transport on the ensemble average, [4] [3].

It is interesting to notice that the local velocity field $\vec{v}(\vec{x}, t)$ obeys the natural (local) momentum conservation law which directly originates from the rules of the Itô calculus for Markovian diffusion processes, [1], and from the first moment equation in the diffusion approximation (!) of the Kramers theory, [4]:

$$
\partial_{t} \vec{v}+(\vec{v} \cdot \vec{\nabla}) \vec{v}=\vec{\nabla}(\Omega-Q) .
$$

An effective potential function $\Omega(\vec{x})$ can be expressed in terms of the forward drift $\vec{b}(\vec{x})=\frac{\vec{F}(\vec{x})}{m \beta}$ as follows: $\Omega=\frac{\vec{F}^{2}}{2 m^{2} \beta^{2}}+\frac{D}{m \beta} \vec{\nabla} \cdot \vec{F}$.

Let us emphasize that it is the diffusion (Smoluchowski) approximation which makes the right-hand-side of Eq. (3) substantially different from the usual moment equations appropriate for the Brownian motion. In particular, the force $\vec{F}$ presumed to act upon an individual particle, does not give rise in Eq. (3) to the expression $-\frac{1}{m} \vec{\nabla} V$ which might be expected on the basis of kinetic theory intuitions and moment identities directly derivable from the Karmers equation, but to the term $+\vec{\nabla} \Omega$.

Moreover, instead of the standard pressure term, there appears a contribution from a probability density $\rho$-dependent potential $Q(\vec{x}, t)$. It is given in terms of the so-called osmotic velocity field $\vec{u}(\vec{x}, t)=D \vec{\nabla} \ln \rho(\vec{x}, t)$, (cf. [回] $): Q(\vec{x}, t)=\frac{1}{2} \vec{u}^{2}+D \vec{\nabla} \cdot \vec{u}$ and is generic to a local momentum conservation law respected by isothermal Markovian diffusion processes, cf. [四]- [8].

The Smoluchowski drift does not refer to any flows in the noise carrier. To analyze perturbations of the medium and then the resulting intrinsic (mean) flows, a more general function $\vec{b}(\vec{X}(t), t)$, must replace the Smoluchowski drift in Eqs. (1), (2). Forward drifts modify additively the pure noise (Wiener process entry) term in the Itô equations. Under suitable restrictions, we can relate probability measures corresponding to different (in terms of forward drifts !) Fokker-Planck equations and processes by means of the Cameron- 
Martin-Girsanov theory of measure transformations. The Radon-Nikodym derivative of measures is here involved and for suitable forward drifts which are are gradient fields that yields, [ [1], the most general form of an auxiliary potential $\Omega(\vec{x}, t)$ in Eq. (3):

$$
\Omega(\vec{x}, t)=2 D\left[\partial_{t} \phi+\frac{1}{2}\left(\frac{\vec{b}^{2}}{2 D}+\vec{\nabla} \cdot \vec{b}\right)\right] .
$$

We denote $\vec{b}(\vec{x}, t)=2 D \vec{\nabla} \phi(\vec{x}, t)$.

Eq. (4) is a trivial identity, if we take for granted that all drifts are known from the beginning, like in case of typical Smoluchowski diffusions where the external force $\vec{F}$ is a priori postulated. We can proceed otherwise and, on the contrary, one can depart from a suitably chosen space-time dependent function $\Omega(\vec{x}, t)$. From this point of view, while developing the formalism, one should decide what is a quantity of a primary physical interest: the field of drifts $\vec{b}(\vec{x}, t)$ or the potential $\Omega(\vec{x}, t)$.

Mathematical features of the formalism appear to depend crucially on the properties (like continuity, local and global boundedness, Rellich class) of the potential $\Omega$, see e.g. $\llbracket$, 9]. Let us consider a bounded from below (local boundedness from above is useful as well), continuous function $\Omega(\vec{x}, t)$, cf. [4]). Then, by means of the gradient field ansatz for the diffusion current velocity $\left(\vec{v}=\vec{\nabla} S \rightarrow \partial_{t} \rho=-\vec{\nabla} \cdot[(\vec{\nabla} S) \rho]\right)$ we can transform the momentum conservation law (3) of a Markovian diffusion process to the universal Hamilton-Jacobi form:

$$
\Omega=\partial_{t} S+\frac{1}{2}|\vec{\nabla} S|^{2}+Q
$$

where $Q(\vec{x}, t)$ was defined before. By applying the gradient operation to Eq. (5) we recover (3). In the above, the contribution due to $Q$ is a direct consequence of an initial probability measure choice for the diffusion process, while $\Omega$ via Eq. (4) does account for an appropriate forward drift of the process. 
Thus, in the context of Markovian diffusion processes, we can consider a closed system of partial differential equations which comprises the continuity equation $\partial_{t} \rho=-\vec{\nabla}(\vec{v} \rho)$ and the Hamilton-Jacobi equation (5), plus suitable initial (and/or boundary) data. The underlying diffusion process is specified uniquely, cf. [4, 5].

Since the pertinent nonlinearly coupled system equations looks discouraging, it is useful to mention that a linearisation of this problem is provided by a time-adjoint pair of generalised diffusion equations in the framework of the so-called Schrödinger boundary data problem, [4, 5]. The standard heat equation appears as a very special case in this formalism.

The local conservation law (3) acquires a direct physical meaning (the rate of change of momentum carried by a locally co-moving with the flow volume, [4]), only if averaged with respect to $\rho(\vec{x}, t)$ over a simply connected spatial area. If $V$ stands for a volume enclosed by a two-dimensional outward oriented surface $\partial V$, we define a co-moving volume on small time scales, by deforming the boundary surface in accordance with the local current velocity field values. Then, let us consider at time $t$ the displacement of the boundary surface $\partial V(t)$ defined as follows: $\vec{x} \in \partial V \rightarrow \vec{x}+\vec{v}(\vec{x}, t) \triangle t$ for all $\vec{x} \in \partial V$. Up to the first order in $\Delta t$ this guarantees the conservation of mass (probability measure) contained in $V$ at time $t$ i. e. $\int_{V(t+\triangle t)} \rho(\vec{x}, t+\triangle t) d^{3} x-\int_{V(t)} \rho(\vec{x}, t) d^{3} x \sim 0$.

The corresponding (to the leading order in $\triangle t$ ) quantitative momentum rate-of-change measure reads, cf. [4], $\int_{V} \rho \vec{\nabla}(\Omega-Q) d^{3} x$.

For a particular case of the free Brownian expansion of an initially given $\rho_{0}(\vec{x})=\frac{1}{\left(\pi \alpha^{2}\right)^{3 / 2}} \exp \left(-\frac{x^{2}}{\alpha^{2}}\right)$, where $\alpha^{2}=4 D t_{0}$, we would have $-\int_{V} \rho \vec{\nabla} Q d^{3} x=$ $-\int_{\partial V} P d \vec{\sigma}$, where $Q(\vec{x}, t)=\frac{\vec{x}^{2}}{8\left(t+t_{0}\right)^{2}}-\frac{3 D}{2\left(t+t_{0}\right)}$, while the "osmotic pressure" contribution reads $P(\vec{x}, t)=-\frac{D}{2\left(t+t_{0}\right)} \rho(\vec{x}, t)$ for all $\vec{x} \in R^{3}$ and $t \geq 0$.

The current velocity $\vec{v}(\vec{x}, t)=\vec{\nabla} S(\vec{x}, t)=\frac{\vec{x}}{2\left(t+t_{0}\right)}$ is linked to the HamiltonJacobi equation $\partial_{t} S+\frac{1}{2}|\vec{\nabla} S|^{2}+Q=0$ whose solution reads: $S(\vec{x}, t)=$ 
$\frac{\vec{x}^{2}}{4\left(t+t_{0}\right)}+\frac{3}{2} D \ln \left[4 \pi D\left(t+t_{0}\right)\right]$

Let us observe that the initial data $\vec{v}_{0}=-D \vec{\nabla} \ln \rho_{0}=-\vec{u}_{0}$ for the current velocity field indicate that we have totally ignored a crucial preliminary stage of the dynamics on the $\beta^{-1}$ time scale, when the Brownian expansion of an initially static ensemble has been ignited and so particles have been ultimately set in motion.

Notice also that our "osmotic expansion pressure" $P(\vec{x}, t)$ is not positive definite, in contrast to the familiar kinetic theory (equation of state) expression for the pressure $P(\vec{x})=\alpha \rho^{\beta}(\vec{x}), \alpha>0$ appropriate for gases. The admissibility of the negative sign of the "pressure" function encodes the fact that the Brownian evolving concentration of particles generically decompresses (blows up), instead of being compressed by the surrounding medium.

The loss (in view of the "osmotic" migration) of momentum stored in a control volume at a given time, may be here interpreted in terms of an acceleration $-\int_{V} \rho \vec{\nabla} Q d^{3} x$ induced by a fictituous "attractive force".

By invoking an explicit Hamilton-Jacobi connection (5), we may attribute to a diffusing Brownian ensemble the mean kinetic energy per unit of mass $\int_{V} \rho \frac{1}{2} \vec{v}^{2} d^{3} x$. In view of $<\vec{x}^{2}>=6 D\left(t+t_{0}\right)$, we have also $\int_{R^{3}} \rho \frac{1}{2} \vec{v}^{2} d^{3} x=$ $\frac{3 D}{4\left(t+t_{0}\right)}$. Notice that the mean energy $\int_{V} \rho\left(\frac{1}{2} \vec{v}^{2}+Q\right) d^{3} x$ needs not to be positive. Indeed, this expression identically vanishes after extending integrations from $V$ to $R^{3}$. On the other hand the kinetic contribution, initially equal $\int_{R^{3}} \frac{1}{2} \rho v^{2} d^{3} x=3 D / \alpha^{2}$ and evidently coming from nowhere, continually diminishes and is bound to disappear in the asymptotic $t \rightarrow \infty$ limit, when Brownian particles become uniformly distributed in space.

Normally, diffusion processes yielding a nontrivial matter transport (diffusion currents) are observed for a non-uniform concentration of colloidal particles which are regarded as independent (non-interacting). We can however devise a thought (numerical) experiment that gives rise to a corresponding transport in terms of an ensemble of sample (and thus independent) Brown- 
ian motion realisations on a fixed finite time interval, instead of considering a multitude of them (migrating swarm of Brownian particles) simultaneously.

Let us assume that "an effort" (hence, an energy loss/gain which implies local deviations from thermal equilibrium conditions) of the random medium, on the $\beta^{-1}$ scale, to produce a local Brownian diffusion current $(\rho \vec{v})\left(\vec{x}, t_{0}\right)$ out of the initially static ensemble and thus to decompress (lower the blowup tendency) an initial non-uniform probability distribution, results in the effective osmotic reaction of the random medium. This is the Brownian recoil effect of Ref. [2].

In that case, the particle swarm propagation scenario becomes entirely different from the standard Brownian one. First of all, the nonvanishing forward drift $\vec{b}=\vec{u}$ is generated as a dynamical (effective, statistical here !) response of the bath to the enforced by the bath particle transport with the local velocity $\vec{v}=-\vec{u}$. Second, we need to account for a parellel inversion of the pressure effects (compression $+\vec{\nabla} Q$ should replace the decompression $-\vec{\nabla} Q)$ in the respective local momentum conservation law.

Those features can be secured through an explicit realization of the actionreaction principle which we promote to the status of the third Newton law in the mean.

On the level of Eq. (3), once averaged over a finite volume, we interpret the momentum per unit of mass rate-of-change $\int_{V} \rho \vec{\nabla}(\Omega-Q) d^{3} x$ which occurs exclusively due to the Brownian expansion mechanism, to generate a counterbalancing rate-of-change tendency in the random medium. To account for the emerging forward drift and an obvious modification of the subsequent expansion of an ensemble of particles, we re-define Eq. (3) by setting $-\int_{V} \rho \vec{\nabla}(\Omega-Q) d^{3} x$ in its right-hand-side instead of $+\int_{V} \rho \vec{\nabla}(\Omega-Q) d^{3} x$. That amounts to an instantaneous implementation of the third Newton law in the mean (action-reaction principle) in Eq. (3).

Hence, the momentum conservation law for the process with a recoil (where the reaction term replaces the decompressive "action" term) would 
read:

$$
\partial_{t} \vec{v}+(\vec{v} \cdot \vec{\nabla}) \vec{v}=\vec{\nabla}(Q-\Omega)
$$

so that

$$
\partial_{t} S+\frac{1}{2}|\vec{\nabla} S|^{2}-Q=-\Omega
$$

stands for the corresponding Hamilton-Jacobi equation, cf. [5, 10], instead of Eq. (5). A suitable adjustment (re-setting) of the initial data is here necessary.

In the coarse-grained picture of motion we thus deal with a sequence of repeatable scenarios realised on the Smoluchowski process time scale $\triangle t$ : the Brownian swarm expansion build-up is accompanied by the parallel counterflow build-up, which in turn modifies the subsequent stage of the Brownian swarm migration (being interpreted to modify the forward drift of the process) and the corresponding built-up anew counterflow.

The new closed system of partial differential equations refers to Markovian diffusion-type processes again, [1, 4, 9]. The link is particularly obvious if we observe that the new Hamilton-Jacobi equation (7) can be formally rewritten in the previous form (5) by introducing:

$$
\Omega_{r}=\partial_{t} S+\frac{1}{2}|\vec{\nabla} S|^{2}+Q
$$

where $\Omega_{r}=2 Q-\Omega$ and $\Omega$ represents the previously defined potential function of any Smoluchowski (or more general) diffusion process.

It is $\Omega_{r}$ which via Eq. (4) would determine forward drifts of the Markovian diffusion process with a recoil. They must obey the Cameron-MartinGirsanov identity $\Omega_{r}=2 Q-\Omega=2 D\left[\partial_{t} \phi+\frac{1}{2}\left(\frac{\vec{b}^{2}}{2 D}+\vec{\nabla} \cdot \vec{b}\right)\right]$.

Our new closed system of equations is badly nonlinear and coupled, but its linearisation can be immediately given in terms of an adjoint pair of


$\frac{\Omega}{2 D} \psi$ with a solution $\psi=\rho^{1 / 2} \exp (i S)$ and its complex adjoint makes the job, if we regard $\rho$ together with $S$ to remain in conformity with the previous 
notations. The choice of $\psi(\vec{x}, 0)$ gives rise to a solvable Cauchy problem. (Notice that by setting $D=\frac{\hbar}{2 m}$ we recover the standard quantum mechanical notation.)

This feature we shall exploit in below. Notice that, for time-indepedent $\Omega$, the total energy $\int_{R^{3}}\left(\frac{v^{2}}{2}-Q+\Omega\right) \rho d^{3} x$ of the diffusing ensemble is a conserved quantity.

The general existence criterions for Markovian diffusion processes of that kind, were formulated in Ref. [9], see also [5, 国.

Let us consider a simple one-dimensional example. In the absence of external forces, we solve the equations (in space dimension one) $\partial_{t} \rho=-\nabla(v \rho)$ and $\partial_{t} v+(v \nabla) v=+\nabla Q$, with an initial probability density $\rho_{0}(x)$ chosen in correspondence with the previous free Brownian motion example. We denote $\alpha^{2}=4 D t_{0}$. Then, $\rho(x, t)=\frac{\alpha}{\left[\pi\left(\alpha^{4}+4 D^{2} t^{2}\right)\right]^{1 / 2}} \exp \left[-\frac{x^{2} \alpha^{2}}{\alpha^{4}+D^{2} t^{2}}\right]$ and $b(x, t)=v(x, t)+u(x, t)=\frac{2 D\left(\alpha^{2}-2 D t\right) x}{\alpha^{4}+4 D^{2} t^{2}}$ are the pertinent solutions. Notice that $u(x, 0)=-\frac{2 D x}{\alpha^{2}}=b(x, 0)$ amounts to $v(x, 0)=0$, while in the previous free Brownian case the initial current velocity was equal to $-D \nabla \ln \rho_{0}$. This re-adjustment of the initial data can be interpreted in terms of the counterbalancing (recoil) phenomenon: the would-be initial Brownian ensemble current velocity $v_{0}=-u_{0}$ is here completely saturated by the emerging forward drift $b_{0}=u_{0}$, see e.g. also [2]. We deal also with a fictituous "repulsive" force, which corresponds to the compression (pressure upon) of the Brownian ensemble due to the counter-reaction of the surrounding medium. We can write things more explicitly. Namely, now: $Q(x, t)=\frac{2 D^{2} \alpha^{2}}{\alpha^{4}+4 D^{2} t^{2}}\left(\frac{\alpha^{2} x^{2}}{\alpha^{4}+4 D^{2} t^{2}}-1\right)$ and the corresponding pressure term $\left(\nabla Q=\frac{1}{\rho} \nabla P\right)$ reads $P(x, t)=-\frac{2 D^{2} \alpha^{2}}{\alpha^{4}+4 D^{2} t^{2}} \rho(x, t)$ giving a positive contribution $+\nabla Q$ to the local conservation law. The related Hamilton-Jacobi equation $\partial_{t} S+\frac{1}{2}|\nabla S|^{2}=+Q$ is solved by $S(x, t)=$ $\frac{2 D^{2} x^{2} t}{\alpha^{4}+4 D^{2} t^{2}}-D \arctan \left(-\frac{2 D t}{\alpha^{2}}\right)$. With the above form of $Q(x, t)$ one can readily check that the Cameron-Martin-Girsanov constraint euqation for the forward drift of the Markovian diffusion process with a recoil is automatically valid 
for $\phi=\frac{1}{2} \ln \rho+S: 2 Q=2 D\left[\partial_{t} \phi+\frac{1}{2}\left(\frac{b^{2}}{2 D}+\nabla \cdot b\right)\right]$.

In anology with our free Brownian motion discussion, let us observe that presently $<x^{2}>=\frac{\alpha^{2}}{2}+\frac{2 D^{2} t^{2}}{\alpha^{2}}$. It is easy to demonstrate that the quadratic dependence on time persists for arbitrarily shaped initial choices of the probability distribution $\rho_{0}(x)>0$. That signalizes an anomalous behaviour (enhanced diffusion) of the pertinent Markovian process when $\Omega=0$ i. e. $\Omega_{r}=2 Q$.

We can evaluate the kinetic energy contribution $\int_{R} \rho \frac{v^{2}}{2} d x=\frac{4 D^{4} t^{2}}{\alpha^{2}\left(\alpha^{4}+4 D^{2} t^{2}\right)}$ which in contrast to the Brownian case shows up a continual growth up to the terminal (asymptotic) value $\frac{D^{2}}{\alpha^{2}}$. This value was in turn an initial kinetic contribution in the previous free Brownian expansion example. In contrast to that case, the total energy integral is now finite (finite energy diffusions of Ref. [9]) and reads $\int_{R}\left(\frac{1}{2} v^{2}-Q\right) \rho d x=\frac{D^{2}}{\alpha^{2}}$ (it is a conservation law). The asymptotic value of the current velocity $v \sim \frac{x}{t}$ is twice larger than this appropriate for the Brownian motion, $v \sim \frac{x}{2 t}$.

It is easy to produce an explicit solution to $(7),(8)$ in case of $\Omega(x)=$ $\frac{1}{2} \gamma^{2} x^{2}-D \gamma$, with exactly the same inital probability density $\rho_{0}(x)$ as before. The forward drift of the corresponding diffusion-type process does not show up any obvious contribution from the harmonic Smoluchowski force. It is completely eliminated by the Brownian recoil scenario. One may check that $b(x, 0)=-\frac{2 D x}{\alpha^{2}}=u(x, 0)$, while obviously $b=\frac{F}{m \beta}=-\gamma x$ would hold true for all times, in case of the Smoluchowski diffusion process. Because of the harmonic attraction and suitable initial probability measure choice, we have here wiped out all previously discussed enhanced diffusion features. Now, the dispersion is attentuated and actually the non-dispersive diffusion-type process is realised: $\left\langle x^{2}\right\rangle$ does not spread at all despite of the intrinsically stochastic nature of the dynamics (finite-energy diffusions of Ref. [9]).

It is clear that stationary processes are the same both in case of the standard Brownian motion and the Brownian motion with a recoil. The respective propagation scenarios substantially differ in the non-stationary 
case only.

One may possibly argue, that a sign inversion of the right-hand-side of Eq. (3) which implies (6), can be accomplished by means of an analytic continuation in time (cf. the Euclidean quantum mechanics discussion in Ref. [5]). An examination of free dynamics cases given in the above proves that the third Newton law ansatz is an independent procedure.

Acknowledgements: I would like to thank Professor Eric Carlen for discussion about the scaling limits of the Boltzmann equation and related conservation laws.

\section{References}

[1] E. Nelson, "Dynamical Theories of the Brownian Motion", Princeton, University Press, Princeton, 1967

[2] P. Garbaczewski, J. P. Vigier, Phys. Rev. A 46, (1992), 4634;

[3] P. Garbaczewski, "Perturbations of noise: Origins of isothermal flows", Phys. Rev. E, in press

[4] Ph. Blanchard, P. Garbaczewski, Phys. Rev. E 49, (1994), 3815

[5] J. C. Zambrini, J. Math. Phys. 27, (1986), 2307

[6] B. T. Geilikman, Sov. JETP (Russian edition), 17, (1947), 830

[7] G. A. Skorobogatov, Rus. J. Phys. Chem. 61, (1987), 509

[8] E. Guth, Adv. Chem. Phys. 15, (1969), 363

[9] E. Carlen, Commun. Math. Phys. 94, (1984), 293

[10] P. R. Holland, "Quantum theory of motion", Cambridge University Press, Cambridge, 1993 